\title{
Fluorinated azobenzenes for shape-persistent liquid crystal polymer networks
}

\author{
Supitchaya lamsaard, ${ }^{[a]}$ Emmanuel Anger, ${ }^{[b]}$ Sarah Jane Aßhoff, ${ }^{[a]}$ Alexis Depauw, ${ }^{[a]}$ \\ Stephen P. Fletcher ${ }^{\star,[b]}$ and Nathalie Katsonis ${ }^{*,[a]}$
}

\begin{abstract}
Liquid crystal polymer networks respond with anisotropic deformation to a range of external stimuli. When doped with molecular photo-switches, these networks undergo complex shape modification under illumination. As the deformations reverse when irradiation stops, applications where thermal stability of the activated shape is required were precluded. Previous attempts to incorporate molecular switches with thermally stable photo-isomers were unsuccessful at photo-generating macroscopic shapes that are retained in time. Here, we show that the key to preserving photoactivated molecular deformation on the macroscopic scale is not only to engineer the thermal stability of the photo-switch but also to adjust the cross-linking density in the polymer network and to optimize the molecular orientations in the material. Our strategy resulted in materials containing fluorinated azobenzenes that retain photochemical shape morphing for more than eight days, which constitutes the first demonstration of long-lived photomechanical deformation in liquid crystal polymer networks.
\end{abstract}

Liquid-crystalline polymers containing molecular photo-switches have emerged as a promising and exciting class of materials for achieving optical to mechanical energy conversion. ${ }^{1,2,3}$ The actuation of these smart materials is usually driven by the photoisomerization of azobenzene, because its trans-to-cis isomerization proceeds with a geometrical change at the molecular level that disrupts liquid crystallinity and generates disorder effectively. In response the polymer network deforms anisotropically, with a contraction along the average molecular orientation and expansion orthogonal to it. ${ }^{4}$ Earlier studies have shown the

[a] Prof. dr. N. Katsonis

Bio-inspired and Smart Materials (BNT)

University of Twente

7500AE Enschede, The Netherlands

E-mail: n.h.katsonis@utwente.nl

[b] Prof. dr. S. P. Fletcher

Chemistry Research Laboratories,

University of Oxford,

Mansfield Road, Oxford OX1 3TA, U.K.

E-mail: stephen.fletcher@chem.ox.ac.uk

Supporting information for this article is given via a link at the end of the document. possibility of amplifying the conformational change of azobenzenes to the macroscopic level by integrating these switches into liquid crystal networks. ${ }^{5}$ After irradiation has ceased, shape recovery of these materials is generally observed within minutes or even seconds. ${ }^{6}$ This shape recovery remains difficult to control and constitutes a major hurdle towards practical applications. Shape-persistent liquid crystal polymer networks may give rise to novel materials, ${ }^{7}$ where morphology would be finely controlled by light and preserved in any given shape that could be reached at an intermediate stage of the modus operandi. Such materials would find applications in soft robotics, haptics or biomedical devices, for example as robotic grippers or as components of artificial muscles.

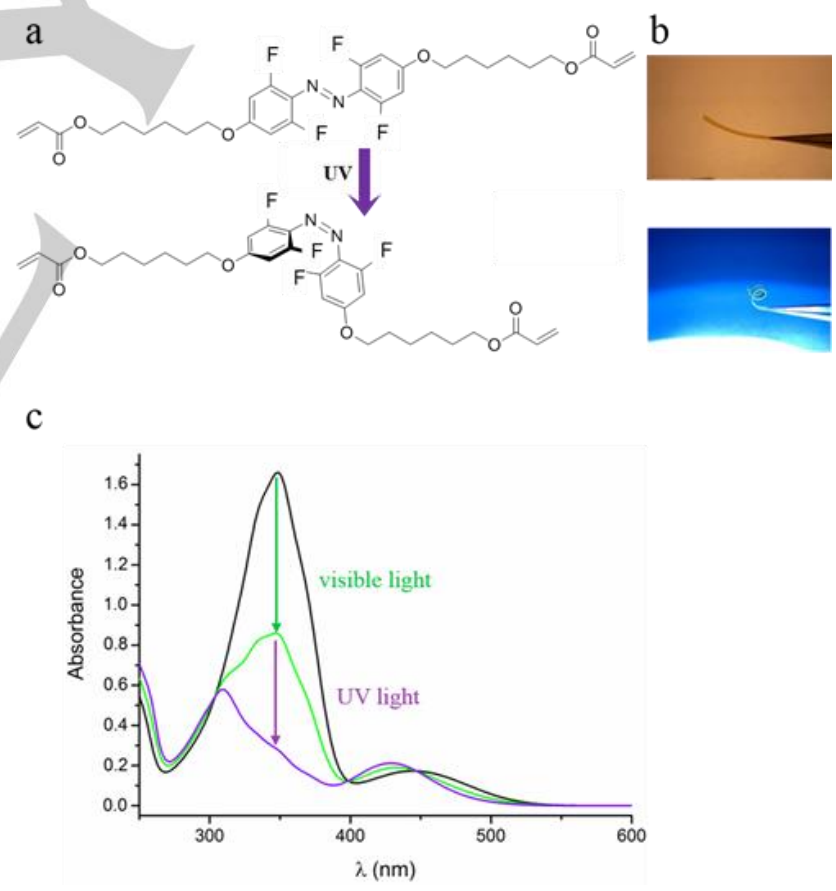

Figure 1. Photo-activation and shape persistence. a) Azo-F photo-isomerizes from its trans- to its cis-form, under irradiation with visible light $(\lambda>420 \mathrm{~nm})$ or UV light $(\lambda=$ $365 \mathrm{~nm})$. b) A polymer ribbon incorporating Azo-F curls under UV irradiation and retains its shape. c) UV-vis absorption spectroscopy shows that Azo-F in dichloromethane switches from trans-to-cis under irradiation. 
The use of ortho-fluorinated azobenzenes as basic switching elements has the potential to increase the lifetime of photogenerated shapes significantly, because the cis-isomer has a half-life of up to two years in solution (Figure 1). ${ }^{8}, 9$ Moreover, halogenated azobenzenes absorb in the visible range and could be switched with sunlight in solution and in liquid crystals. ${ }^{10}$ However, designing general principles to translate molecular switching from the nanoscale up to the functional level remains an open challenge, ${ }^{11}$ and specifically, in some recent examples the (macroscale) shape relaxation seemed decorrelated from the relaxation of a meta-fluorinated azobenzene it incorporated. White et al. have investigated shape changes in a liquid crystal polymer network containing a meta-fluorinated azobenzene. ${ }^{12}$ Despite the cis-form being stable for about $180 \mathrm{~h}$ in solution, as soon as irradiation was ceased, the polymer underwent rapid relaxation to its original shape. The amplitude of deformation was also moderate. This lack of retention in shape-morphing properties was proposed to arise from i) possible interference from hydrogen bonding favoring the trans-form, and ii) the lack of order in the liquid crystal polymer network - implying a random orientation of the switches within the network - which might prohibit effective translation of their shape change into the decrease of order parameter. Here, we propose that, in addition to these contributions, the nature of the coupling between the photoswitch and its environment is a major factor in determining the macroscopic shape-shifting properties of the material. For example, main chain liquid crystal polymers produce stronger thermo-mechanical effects compared to side chain systems. ${ }^{13}$ Other investigations have suggested that combining a low crosslink density with a uniform crosslink distribution is key to designing shapememory polymers. ${ }^{14}$ Thus, we anticipated that the cross-linking density would have to be adjusted. In liquid crystal polymer networks actuated by azobenzene, the crosslinking density reduces the trans-to-cis photoconversion ${ }^{15}$ and attenuates shape memory effects by promoting a faster relaxation of the network. ${ }^{16}$ Moreover, it is known that azobenzene-moieties relax faster when they are strained. ${ }^{17}$ In a highly cross-linked polymer network it is likely that the azobenzenes are strained, especially when a short spacer is used to separate the mesogenic core units from the reactive endgroups. ${ }^{18}$

We designed a liquid crystal polymer network that contains a low ratio of diacrylate cross-linkers and in which the cross-linkers have a longer spacer (Scheme 1). In that network we anticipated that the coupling between the switches and their environment would be sufficiently weak to allow efficient trans-to-cis photo- isomerization of ortho-fluorinated azobenzenes, and strong enough to overcome the elastic properties of the network and control the kinetics that restore the object to its initial shape. Other limiting factors were attenuated or suppressed by diluting the switch within the network down to $5 \mathrm{wt} \%$ of the material only, and by locating the fluorinated switch in the main polymer chain. Recent work on surface morphing has indeed reported that diacrylate azobenzenes used as crosslinkers induce larger amplitude shape changes than azobenzene mono-acrylates. ${ }^{6}$ Our strategy resulted in liquid crystal polymer networks that undergo large photo-induced deformations and that kept their shape for days in any given stage of their operation - i.e. also midway from full conversion.

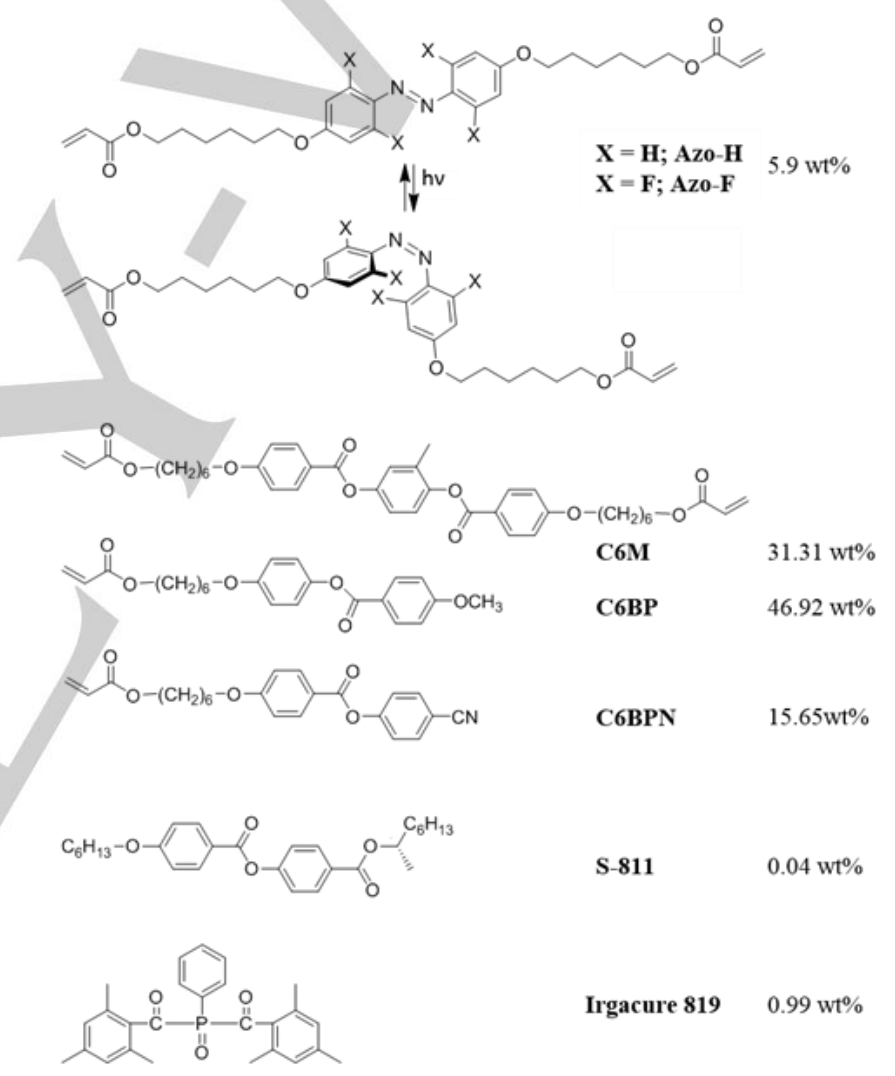

Scheme 1. Photo-switches Azo-H and Azo-F. Two reactive end groups allow incorporating the switches as cross-linkers in the network. In addition to the photo-switches the polymer matrix is built by the reactive mesogens C6M and C6BP, C6BPN bearing two and one polymerizable endgroup respectively. The chiral dopant S-811 was used to achieve a homogeneous left-handed twist in the film and the photoinitiator Irgacure 819 was used to photo-polymerize at $\lambda>$ $420 \mathrm{~nm}$. Other compositions that were tested include the same composition as in [20], however the film displayed poor elastic properties. 

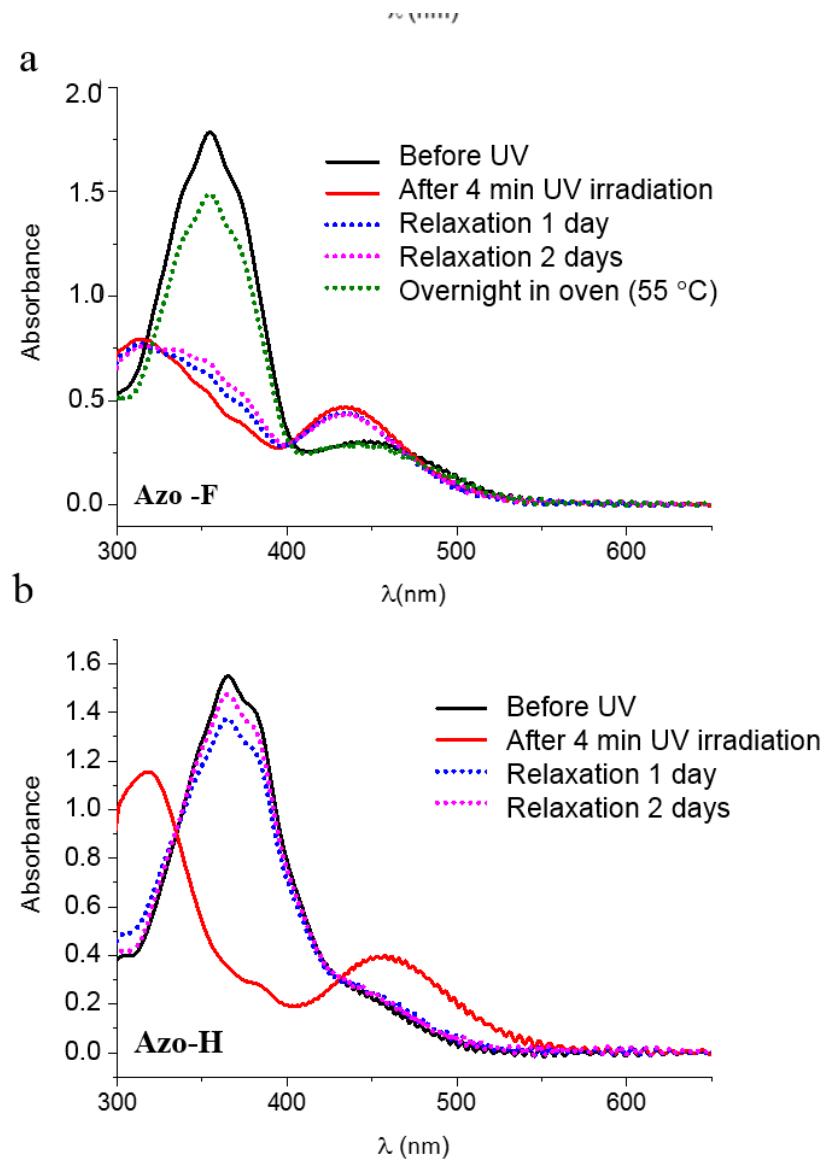

Figure 2. Photo-switching $(\lambda=365 \mathrm{~nm})$ and thermal relaxation of a) Azo-H and b) Azo-F incorporated covalently in thin films of liquid crystal polymer networks. The nominal thickness of the films was $15 \mu \mathrm{m}$ for the spectroscopic investigations.

We synthesized an ortho-fluorinated azobenzene AzoF with two reactive end groups, and its classical counterpart Azo-H for comparison (Scheme 1 and Figure S1 in Supporting Information). A liquid crystal polymer film was prepared by photo-polymerization of a nematic liquid crystal $(\lambda>420 \mathrm{~nm})$ containing reactive monomers, Azo-F or Azo-H, a few percent of photo-initiator (Irgacure 819), and traces of chiral dopant (S-811), in a glass cell promoting a twist geometry for the liquid crystal. A step-by-step procedure describing how to optimize liquid crystal polymer networks to form spring-like macroscopic geometries and the preparation of these materials is reported elsewhere. ${ }^{19,20}$ It should be noted that the use of a twist geometry is particularly suitable for studying shape changes because liquid crystal polymer films with a twisted or a splayed organization display larger amplitude shape shifting than similar films with planar organization of the director. ${ }^{21}$
The photo-isomerization and relaxation of the switches embedded covalently in the liquid crystal polymer film were studied by UV-vis absorption spectroscopy. Comparing their relaxation rates in the dark revealed that the thermal relaxation of cis-Azo-F to trans-Azo-F is slower than that of cis-Azo-H. After two days, the characteristic absorption band of cis-Azo-F remains whereas, within same period, Azo-H embedded in the network showed nearly full conversion of the $c i$-isomer into the trans-isomer (Figure 2). The thermal stability of cis-Azo-F was further confirmed by ${ }^{1} \mathrm{H}-\mathrm{NMR}$ spectroscopy (Figure S2 in Supporting Information).

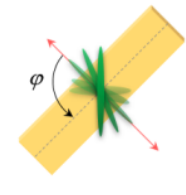

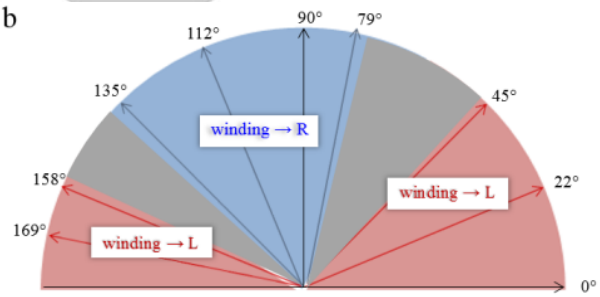

Figure 3. a) The angle $\varphi$ is defined as the angle between the preferential orientation of the molecules at midplane, and the cutting direction. b) Shape and actuation modes of the polymer springs containing Azo-F as a function of the angle between the orientation of the molecules at mid-plane and the cutting direction $\varphi$. The nominal thickness of the ribbons was $50 \mu \mathrm{m}$.

The shape recovery of springs incorporating either Azo$\mathbf{H}$ or Azo-F was investigated. We cut ribbons out of liquid crystal polymer films with a twist-nematic organization, in a cutting direction that is defined by the angle $\varphi$ between the orientation of the molecules at midplane and the cutting direction (Figure 3a). For $\varphi=90^{\circ}$ it is clear that the Azo-F ribbon relaxes slower than the Azo-H ribbon: after two days the Azo-H based film was nearly half way back to its original shape while the film containing Azo-F showed only minor shape changes (Figure 4). However, this comparison was hampered by the fact that the photo-deformation of ribbons incorporating different switches was not the same, despite being characterized by the same angle $\varphi$ (Figure $3 b)$. In previous work we have shown that the shape of the springs is determined by the cutting angle, the handedness of the twist in the cell, and the gradient in cross-linking density. ${ }^{20}$ Here, apart from the switches Azo-F and Azo-H themselves, the films have the same composition. The fact that different springs are formed for the same cutting angle is likely related to the formation of different profiles for the gradient in crosslinking density that runs through the thickness of the sample, as the switches have different concentrations and also different absorption coefficients. 
Consequently we studied the shape recovery of objects that contain either Azo-F or Azo-H and that have identical macroscopic geometries - that means the molecular organization in the ribbons is different, because Azo-F and Azo-H networks produce different shapes. Ribbons with similar macroscopic shapes and deformations were selected from our library of photoresponsive springs (Figure S3 in the Supporting Information). Specifically, we chose ribbons that bend and compared the rate of their backwards bending in the dark (Figure 5). Within a week after irradiation with UV light, a ribbon cut out from a thin film containing Azo$\mathbf{H}\left(\varphi=0^{\circ}\right)$ moved back to its original shape. In contrast, a flat ribbon containing Azo-F $\left(\varphi=112.5^{\circ}\right)$ transforms into a bended shape under illumination with UV light, and retains that shape for at least eight days, i.e. the photo-generated shapes of Azo-F display long-lived persistence.

For a spring cut at $\varphi=45^{\circ}$, we observe a time-delay before the full amplitude of the photo-mechanical response of the Azo-F network is reached, that has not been reported in earlier investigations. Specifically, comparing Fig. 5iii and Fig. 5v, we note that the material incorporating Azo-F continues bending, even after UV irradiation was ceased.

The system features another kinetic difference between the dynamic behavior of Azo-H and Azo-F once incorporated into the network and the macroscopic shape relaxation. Specifically, the shape relaxation of the liquid crystal network is slower than the cis-to-trans relaxation of Azo-F i.e., overall the material relaxes even slower than the fluorinated azobenzenes it incorporates (Figure $4 \mathrm{~b}$ and $4 \mathrm{c}$ ). Recent observations have reported a kinetic lag also in the surface morphing of liquid crystal elastomers. Specifically, a kinetic mismatch was observed between the slower azobenzene relaxation and the faster relaxation of the polymer network, in systems where the relaxation rate is dominated by the viscoelastic deformation of the polymer network rather than by the photochemistry of the switch. ${ }^{6}$ Here, Azo-F responds quicker than the liquid crystal network in which it is incorporated, both during the photo-step and during relaxation. We anticipate that this behavior arises from a lag in the gradual reorientation and subsequent reorganization of the polymer in which the cis-Azo-F is embedded.

In summary, incorporating Azo-F covalently into liquid crystal polymer networks as switchable cross-linkers, we found that these dynamic units performed well in transducing light into complex mechanical function and that the shape of the Azo-F network displayed longlived (> 8 days) shape persistence. The composition of the polymer matrix was key in preserving photo- activated molecular deformation on the macroscopic scale. We also show that for some compositions and geometries, the response of the polymer network lags the molecular switching, in both directions, which shows similarities with a hysteresis behavior. The work finally suggests possible developments towards responsive materials displaying phototropism, because in solution, ortho-fluorinated switches can also be activated by visible light (Figure 1). ${ }^{8}$ Overall these results demonstrate that molecular photo-switches can be engineered to overcome thermal relaxation, and that the coupling between these switches and their environment remains key to harnessing molecular photo-switching when designing soft machines by transduction of light into mechanical work.

\section{Experimental Section}

The synthesis of Azo-H and Azo-F is described in the supporting information. The liquid crystal monomers were purchased from Synthon Chemicals. The chiral dopant S-811 and the photo-initiator were supplied by Sigma-Aldrich. HPLC grade dichloromethane was used for preparation of the liquid crystal mixture. Liquid crystal cells were purchased from E.H.C. Co., Ltd, Japan.

The liquid crystalline base composition mixture contains (mol \%): 4.2\% azo-switch monomer (either Azo-F or Azo-H); $94.7 \%$ liquid crystal monomers (including C6M:C6BP:C6BPN in molar ratio 1.17 : 2.96 : 1$) ; 0.038 \%$ chiral dopant (S-811); and, $1.1 \%$ photo-initiator (Irgacure 819). The amount of chiral dopant S-811 was adjusted to induce a $50 \mu \mathrm{m}$ quarter pitch. All components were brought together by dissolution in dichloromethane and further the solvent was evaporated at $60{ }^{\circ} \mathrm{C}$ under a nitrogen stream. After removal of solvent, the liquid crystal was heated up to isotropic phase $\left(80^{\circ} \mathrm{C}\right)$ for $30 \mathrm{~min}$ gradually cooled down to the nematic phase $\left(48^{\circ} \mathrm{C}\right)$, injected in the liquid crystal cell and left at $48{ }^{\circ} \mathrm{C}$ for $1 \mathrm{~h}$, before photopolymerisation with visible light for $3 \mathrm{~h}$, using an Edmund MI-150 high-intensity illuminator equipped with a cut-off filter $\left(\lambda \geq 420 \mathrm{~nm}, 77-170 \mathrm{~mW} . \mathrm{cm}^{-2}\right)$. The polymerized film was retained in the cell at $55{ }^{\circ} \mathrm{C}$ overnight to promote full polymerisation.

The nominal gap of the twist cells was $50 \mu \mathrm{m}$ and the nominal gap of the planar cells was $15 \mu \mathrm{m}$, respectively. The ribbons were cut with a scalpel from twist cells. Their typical dimensions were $\sim 1 \mathrm{x} \sim 6 \mathrm{x} 0.05 \mathrm{~mm}$. 
UV/vis absorption spectra were recorded using a Perkin Elmer Lambda 850 UV-vis spectrophotometer. Shape changes were captured with a Dino-Lite Pro AM4113T USB microscope.

[1] Z. Mahimwalla, K. G. Yager, J-I. Mamiya, A. Shishido, A. Priimagi, C. J. Barrett Polym. Bull. 2012, 69, 967-1006.

[2] T. J. White, D. J. Broer, Nat. Mater. 2015, 14, 1087.

[3] L. T. de Haan, A. P. H. J. Schenning, D. J. Broer, Polymer 2014, 55, 5885.

[4] K. D. Harris, R. Cuypers, P. Scheibe, C. van Oosten, C. W. M. Bastiaansen, J. Lub, D. J. Broer, J. Mater. Chem. 2005, 15, 5043.

[5] a) Y. Yu, M. Nakano, T. Ikeda, Nature 2003, 425, 145; b) M. Lopez, H. Finkelmann, P. Palffy-Muhoray, M. Shelley, Nat. Mater. 2004, 3, 307-310; c) D. Liu, C. W. M. Bastiaansen, J. M. J. Den Toonder, D. J. Broer, Angew. Int. Ed. 2012, 51, 892-896; d) A. Bobrovsky, V. Shibaev, M. Cigl, V. Hamplova, F. Hampl, G. Elyashevitch, J. Mater. Chem. C 2014, 2, 4482-4489.

[6] D. Liu, D. J. Broer, Nat. Commun. 2015, 6, 8334 doi: 10.1038/ncomms9334.

[7] G. J. Berg, M. K. McBride, C. Wang, C. N. Bowman, Polymer 2014, 55, 5849-5872.

[8] D. Bléger, J. Schwarz, A. M. Brouwer, S. J. Hecht, J. Am. Chem. Soc. 2012, 134, 20597.

[9] For integration of fluorinated azobenzenes in other complex molecular systems see also a) A. Rullo, A. Reiner, A. Reiter, D. Trauner, E. Y. Isacoff, G. A. Woolley, Chem. Commun. 2014, 50, 14613; b) O. S. Bushuyev, A. Tomberg, T. Friščić, C. J. Barrett, J. Am. Chem. Soc. 2013, 135, 12556.

[10] A. Bobrovsky, V. Shibaev, M. Cigl, V. Hamplova, F. Hampl, G. Elyashevitch, J. Mater. Chem. C 2014, 2, 4482.

\section{Acknowledgements}

This work was supported financially by the European Research Council (Starting Grant 307784 to N.K.), the Netherlands Organization for Scientific Research (Vidi Grant to N.K.) and the EPSRC (Standard Grant EP/M002144/1 to S.P.F.).

Keywords: smart materials $\bullet$ molecular switches $\bullet$ soft actuators $\bullet$ liquid crystals $\bullet$ photochromism

[11] a) M. Peplow, Nature 2015, 525, 18; b) J. M. Abendroth, O. S. Bushuyev, P. S. Weiss, C. J. Barrett, ACS Nano 2015, 9, 7746

[12] K. Min Lee, B. M. Lynch, P. Luchette, T. J. White, J. Pol. Sci. A 2014, 52, 876.

[13] S. Krause, F. Zander, G. Bergmann, H. Brandt, H. Wertmer, H. Finkelmann, C. R. Chimie 2009, 12, 85.

[14] H. Koerner, R. J. Strong, M. L. Smith, D. H. Wang, L.S. Tan, K. M. Lee, T. J. White, R. A. Vaia Polymer 2013, 54, 391-402.

[15] Y. Yu , M. Nakano, A. Shishido, T. Shiono, T. Ikeda Chem. Mater. 2004, 16, 1637-1643.

[16] L. Cheng, Y. Torres, K. Min Lee, A. J. McClung, J. Baur, T. J. White, W. S. Oates, J. Appl. Phys. 2012, 112, 013513.

[17] C. Barrett, A. Natansohn, P. Rochon, Chem. Mater. 1995, 7, 899-903.

[18] J. Garcia-Amorós, H. Finkelmann, D. Velasco, J. Mater. Chem. 2011, 21, 1094.

[19] S. Iamsaard, S. J. Aßhoff, F. Lancia, S. P. Fletcher, N. Katsonis, Nat. Protoc. 2016, in press.

[20] S. Iamsaard, S. J. Aßhoff, B. Matt, T. Kudernac, J. J. L. M. Cornelissen, S. P. Fletcher, N. Katsonis, Nat. Chem. 2014, $6,229$.

[21] C. L. van Oosten , K. D. Harris, C. W. M. Bastiaansen, D. J. Broer, Eur. Phys. J. E 2007, 3, 329-336. 
Figure 4. Irradiation and thermal relaxation of ribbons cut at an angle $\varphi=90^{\circ}$, out of a thin film containing a) Azo-H or b) Azo-F as an active switching unit. The percentages of shape recovery are specified at the bottom left corner of the pictures. c) UV-vis absorption spectra and percentage of trans-recovery of the Azo-F switch in the ribbon.

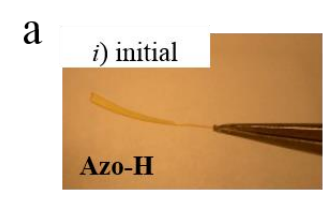

$\mathrm{b}$

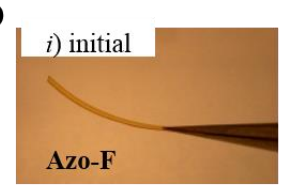

。

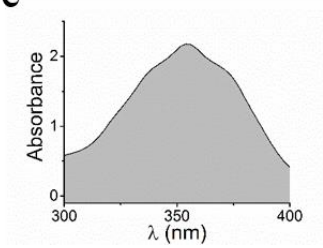

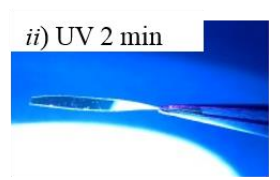
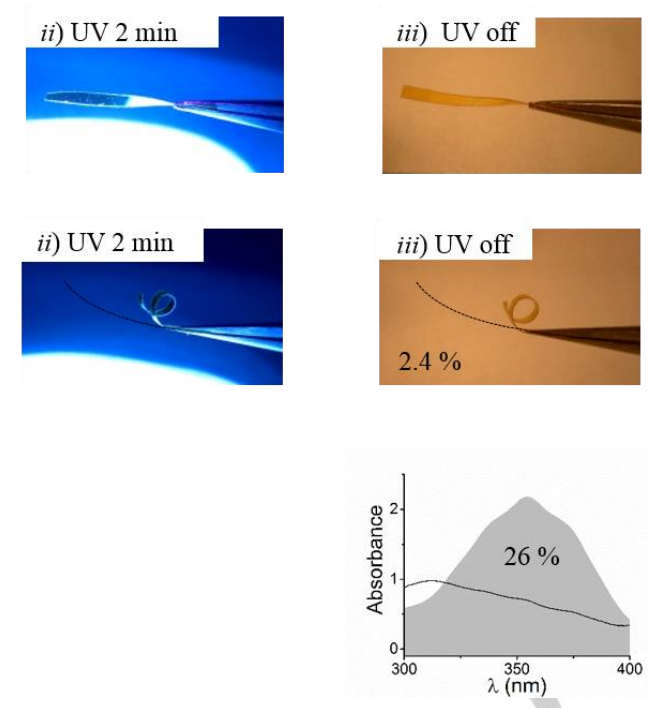
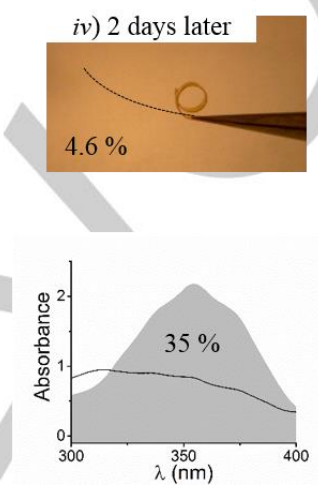
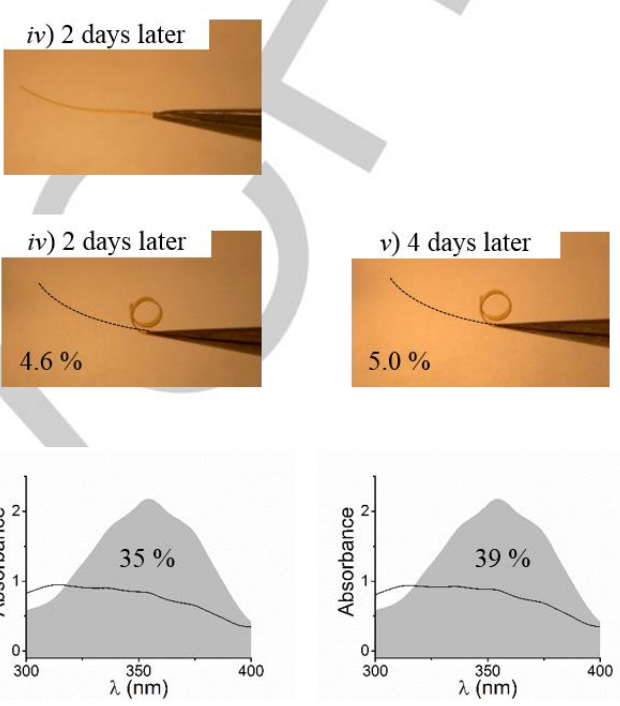

Figure 5. Irradiation and thermal relaxation of ribbons cut from a film containing a) Azo-H or b) Azo-F. The cutting angle is chosen in such a way that the ribbons are flat initially, and bend under irradiation with light. The nominal thickness of the ribbons was $50 \mu \mathrm{m}$.
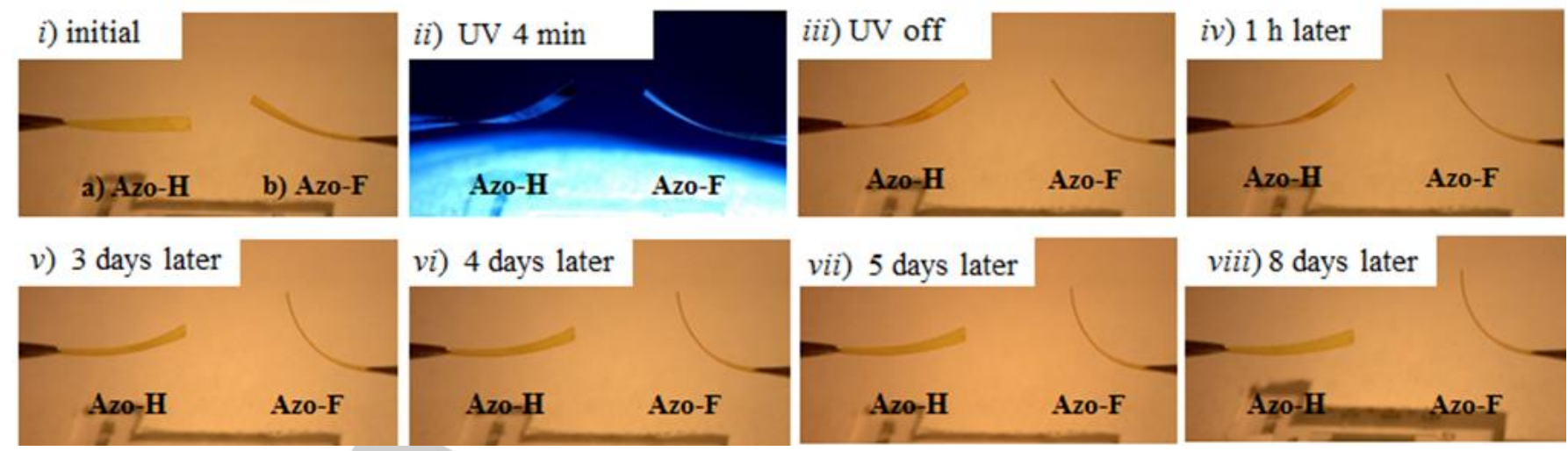

viii) 8 days later

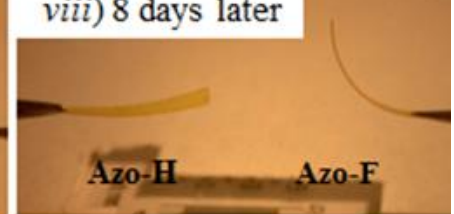


WILEY-VCH

\section{COMMUNICATION}

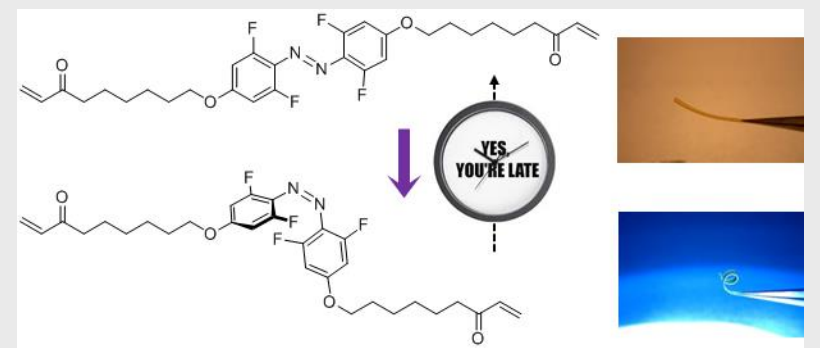

Supitchaya lamsaard, Emmanuel Anger, Sarah Jane Aßhoff, Alexis Depauw, Stephen P. Fletcher* and Nathalie Katsonis*

Page No. - Page No.

Fluorinated azobenzenes for shapepersistent liquid crystal polymer networks 